\title{
The Value of ABO Blood Group and Complete Blood Count for the Prognosis Analysis of Gastric Cancer Patients
}

This article was published in the following Dove Press journal: OncoTargets and Therapy

\author{
Xiao-jie Sun ${ }^{1}, *$ \\ Le $\mathrm{Ai}^{2}$,* \\ Yang-chun Feng (iD) ${ }^{2}$ \\ 'Blood Transfusion Center, Tumor \\ Hospital Affiliated to Xinjiang Medical \\ University, Xinjiang, People's Republic of \\ China; ${ }^{2}$ Clinical Laboratory Center, \\ Tumor Hospital Affiliated to Xinjiang \\ Medical University, Xinjiang, People's \\ Republic of China
}

*These authors contributed equally to this work
Correspondence: Yang-chun Feng Tel +86-99I-78I-9420

Email paopao1987I23@I63.com
Objective: To investigate the value of $\mathrm{ABO}$ blood group and complete blood count results in predicting the survival rate of patients with gastric cancer.

Patients and Methods: A retrospective study was conducted to collect 488 gastric cancer patients diagnosed in the Tumor Hospital Affiliated to Xinjiang Medical University from January 2010 to December 2011. Relevant clinical data were collected by the medical record system, and the patients were followed up by the medical record follow-up system of the hospital. The follow-up was ended until the death of the patients, and the survival time of all patients was obtained. Survival curve and Cox regression analysis model were used to study the role of various indicators in the prognosis of gastric cancer patients.

Results: Neutrophil lymphocyte ratio (NLR), neutrophil monocyte ratio (NMR), lymphocyte monocyte ratio (LMR) and platelet distribution width (PDW) in blood routine test could predict the death outcome of gastric cancer patients, with the predicted thresholds of 1.95 , $13.49,5.22$ and 11.25, respectively. Survival curve analysis showed that female patients, type O blood patients, LMR $>5.22$ patients, NMR $>13.49$ patients and NLR $\leq 1.95$ patients had longer survival. Multivariate Cox regression analysis model showed that gender and NLR were independent prognosis risk factors for gastric cancer, with HR values of 2.964 (95\% CI of 2.258-3.891) and 1.103 (95\% CI of 1.028-1.183), respectively. PLT and PDW were independent prognosis protective factors for gastric cancer, with HR values of 0.998 (95\% CI of $0.997-1.000)$ and 0.891 (95\% CI of $0.797-0.996)$, respectively. Compared with type O blood patients, patients with type A blood, type B blood and type AB blood had 3.472 times (95\% CI 2.562-4.706), 3.368 times (95\% CI 2.454-4.624) and 4.407 times $(95 \%$ CI 2.871-6.766) increased risk of death.

Conclusion: The results of NLR, PLT, PDW and ABO blood group can help to predict the survival of gastric cancer patients.

Keywords: ABO blood group, complete blood count, gastric cancer, prognostic analysis

\section{Introduction}

Gastric cancer includes various tumors that occur in the stomach. More than $95 \%$ of these tumors come from epithelial cells, which named gastric cancer. There are also rare non-epithelial tumors in the stomach, including lymphoma and stromal tumors. In China, gastric cancer as the third highest incidence and the second highest death rate of malignant tumors has caused great damage to public health. ${ }^{1}$ According to the literature, if early gastric cancer is limited to mucosa, the 5-year survival rate is about $90 \%$; If only submucosal cancer is involved, the 5 -year survival rate is about $85 \%$; however, if 
gastric cancer is in middle, late stage or advanced stage, the 5-year survival rate is less than $40 \%{ }^{2,3}$ The clinical treatment of gastric cancer includes surgery, chemotherapy, targeted therapy, supportive treatment, etc. After receiving these treatment regimens, how to evaluate the prognosis of patients has important clinical significance for clinicians and patients, which can help clinicians to change the treatment regimens and improve the survival confidence of patients.

Complete Blood Count (CBC) is one of the most common Clinical laboratory examination project. Absolute counts of Neutrophils, Lymphocytes and Monocytes reflect the inflammatory response and the body's integral immune status. Inflammatory markers indicating prognosis in peripheral blood include the ratio of neutrophils to lymphocytes (NLR), the ratio of lymphocytes to monocytes (LMR), the ratio of platelets to lymphocytes (PLR), red blood cells distribution width (RDW) and platelet distribution width (PDW), these indicators have been proven to be closely related to the prognosis of a variety of cancers. ${ }^{4,5} \mathrm{ABO}$ blood group also has been explored to be closely related to the prognosis of various malignant tumors. ${ }^{6}$ In clinical laboratories, the tests of $\mathrm{CBC}$ and $\mathrm{ABO}$ blood groups are inexpensive and required for every patients, so experimental data are relatively easy to obtain. Therefore, it has great clinical significance by the results of $\mathrm{ABO}$ blood group and $\mathrm{CBC}$ to predict the prognosis of gastric cancer patients.

\section{Patients and Methods}

\section{Data Source}

This retrospective study was approved by the ethics committee of Tumor Hospital Affiliated to Xinjiang Medical University, this study only used existing data of inpatients, and no interventions or injuries to patients, and all patient data confidentiality and compliance with the Declaration of Helsinki. So the patient consent to review their medical records was not required by the ethics committee of the tumor hospital affiliated to Xinjiang Medical University. The medical records of gastric cancer patients were allowed to be collected and analyzed. All the cases of gastric cancer were diagnosed by pathological results. A total of 488 cases gastric cancer were collected, including 281 males and 207 females in the Tumor Hospital Affiliated to Xinjiang Medical University from January 2010 to December 2011. The average age is 61 for men and 65 for women. At the same time, the results of the first $\mathrm{CBC}$ test and $\mathrm{ABO}$ blood type test on the first admission were collected, and the patients were followed up by the hospital's case follow-up system. The outcome of the follow-up was the death of patients, and then obtained the survival time of all the patients.

\section{Statistical Analysis}

The area under the curve (AUC) was calculated by the receiver operating characteristic curve (ROC) and the optimal cutoff value of the continuous variable was calculated. The survival curves were compared by Kaplan-Meier method and Logrank test. In addition, univariate and multivariate analyses were performed using Cox regression models to evaluate the effects of multiple covariates on survival outcomes. For all analyses, $\mathrm{P}<0.05$ was defined as significant. SPSS19.0 (SPSS, Chicago, IL) was used for statistical analysis.

\section{Result}

\section{The General Clinical Features of 488 Gastric Cancer Patients}

From Table 1, we can see that among the 488 gastric cancer patients, the proportion of male patients was significantly

Table I Analysis of 488 Gastric Cancer Patients

\begin{tabular}{|c|c|c|}
\hline & $\begin{array}{l}\text { Number of } \\
\text { Cases }\end{array}$ & $\begin{array}{l}\text { Constituent Ratio } \\
\text { (\%) }\end{array}$ \\
\hline \multicolumn{3}{|l|}{ Gender } \\
\hline Male & 281 & 57.58 \\
\hline Female & 207 & 42.42 \\
\hline \multicolumn{3}{|l|}{ Age } \\
\hline$<60$ years old & 169 & 34.63 \\
\hline$\geq 60$ years old & 319 & 65.37 \\
\hline \multicolumn{3}{|l|}{$\begin{array}{l}\text { Helicobacter pylori } \\
\text { infection }\end{array}$} \\
\hline Infection & 310 & 63.52 \\
\hline No infection & 178 & 36.48 \\
\hline \multicolumn{3}{|l|}{ TNM staging } \\
\hline $\mathrm{I} / \mathrm{II}$ & 256 & 52.46 \\
\hline $\mathrm{III} / \mathrm{IV}$ & 232 & 47.54 \\
\hline \multicolumn{3}{|l|}{ CEA } \\
\hline$<5 \mathrm{ng} / \mathrm{mL}$ & 340 & 69.67 \\
\hline$\geq 5 \mathrm{ng} / \mathrm{mL}$ & 148 & 30.33 \\
\hline \multicolumn{3}{|l|}{ CA199 } \\
\hline$<37 \mathrm{U} / \mathrm{mL}$ & 388 & 79.51 \\
\hline$\geq 37 \mathrm{U} / \mathrm{mL}$ & 100 & 20.49 \\
\hline \multicolumn{3}{|l|}{ Blood type } \\
\hline Type O & 217 & 44.47 \\
\hline Type A & 133 & 27.25 \\
\hline Type B & 100 & 20.49 \\
\hline Type AB & 38 & 7.79 \\
\hline
\end{tabular}


higher than female patients, and the proportion of patients over 60 years old was as high as $65.37 \%$, and the infection rate of helicobacter pylori was as high as $63.52 \%$. The positive rates of CEA and CA199, respectively, were $30.33 \%$ and $20.49 \%$. Among the four blood groups of $\mathrm{ABO}$, the detection rate of type $\mathrm{O}$ was the highest, up to $44.47 \%$

\section{The Critical Value of Each Detection Index}

Taking the death of the patient as the final event. The ROC curve was used to find out whether there were appropriate critical values for every test item to predict the death outcome of the patients, so as to prove the significance of various indicators in the prognostic judgment. The quantitative indicators related to the outcome of the patient's death were respectively NLR, NMR, LMR and PDW. The ROC curves and related statistical results of various indicators are shown in Figure 1 and Table 2.

\section{Survival Analysis Results of Every Significant Test Indicators}

Using the survival curve to analyze the significance of gender, blood type and four quantitative indexes relating to the death outcome of gastric cancer patients to judge the prognosis of gastric cancer patients. The results are shown in Figure 2. It could be seen from Figure 2 that the survival period of female patients was longer than male

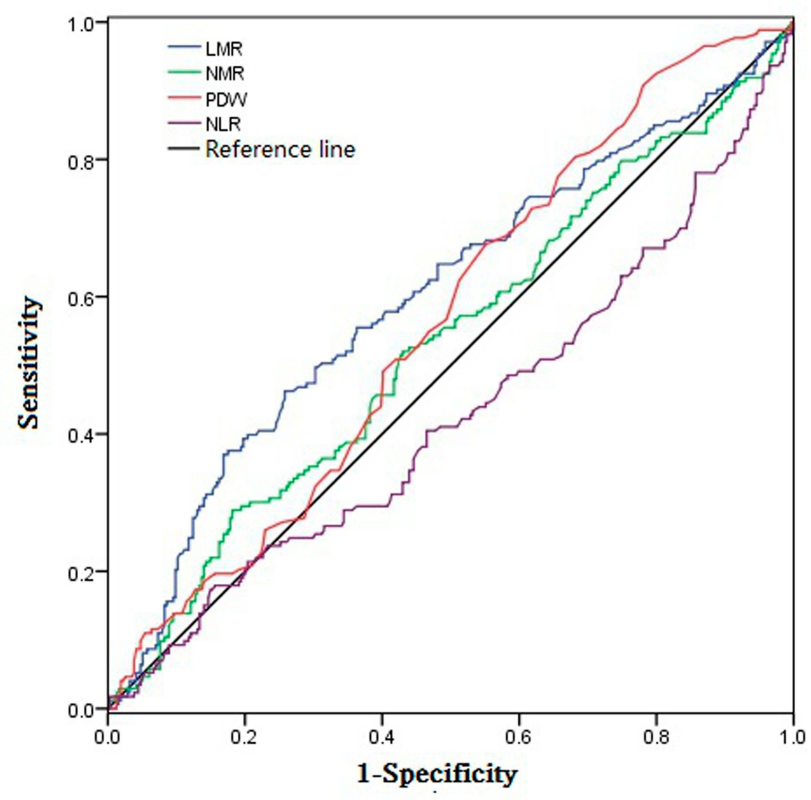

Figure I ROC analysis diagram of meaningful quantitative indicators.
Table 2 ROC Analysis Results of Significant Quantitative Indicators

\begin{tabular}{|l|l|l|l|}
\hline Index & AUC & P value & Critical Value \\
\hline NLR & 0.572 & $0.009^{*}$ & 1.95 \\
NMR & 0.532 & $0.039^{*}$ & 13.49 \\
LMR & 0.601 & $<0.001^{*}$ & 5.22 \\
PDW & 0.563 & $0.021^{*}$ & 11.25 \\
\hline
\end{tabular}

Note: *It has statistical significance.

patients $(\mathrm{P}<0.05)$; the survival period of O-type blood patients was longer than other three types of non-O type blood patients $(\mathrm{P}<0.05)$; Compared to $\mathrm{LMR} \leq 5.22$ group and NMR $\leq 13.49$ group, in LMR >5.22 group and NMR $>13.49$ group, the patients' survival period were longer $(\mathrm{P}<0.05)$, while NLR $\leq 1.95$ group was longer than that of NLR $>1.95$ group $(\mathrm{P}<0.05)$.

\section{Univariate Cox Regression Analysis of Every Indicator}

Univariate Cox regression analysis was used to calculate the value of gender, blood type and every quantitative indicator for the prognosis of gastric cancer (Table 3). It could be found that gender, blood type and NLR were the prognostic factors of gastric cancer. The risk of death in male patients was 1.825 times higher than female patients (95\% CI 1.443-2.308). Compared to type O blood, patients with type A blood had a 2.107-fold increased risk of death (95\% CI 1.536-2.648), the death risk of patients with type B blood increased by 2.094 times (95\% CI 1.558-2.814), and the death risk of patients with type AB blood increased 2.372 times (95\% CI 1.585-3.550). When the NLR value increased by 1 , the risk of death increased by 1.062 times (95\% CI 1.006-1.120).

\section{Multivariate Cox Regression Analysis of Various Indicators}

Multivariate Cox regression analysis was used to calculate the value of gender, blood type and every quantitative indicator on the prognosis of gastric cancer (Table 4). It could be found that gender, blood type and NLR were independent risk factors for the prognosis of gastric cancer. The risk of death in male patients was 2.964 times higher than female patients (95\% CI 2.258-3.891). Compared with type $\mathrm{O}$ blood, the risk of death was 3.472 times higher in patients with type A blood $(95 \%$ CI 2.562-4.706), that of patients with type B blood increased by 3.368 times (95\% CI 2.454-4.624), 

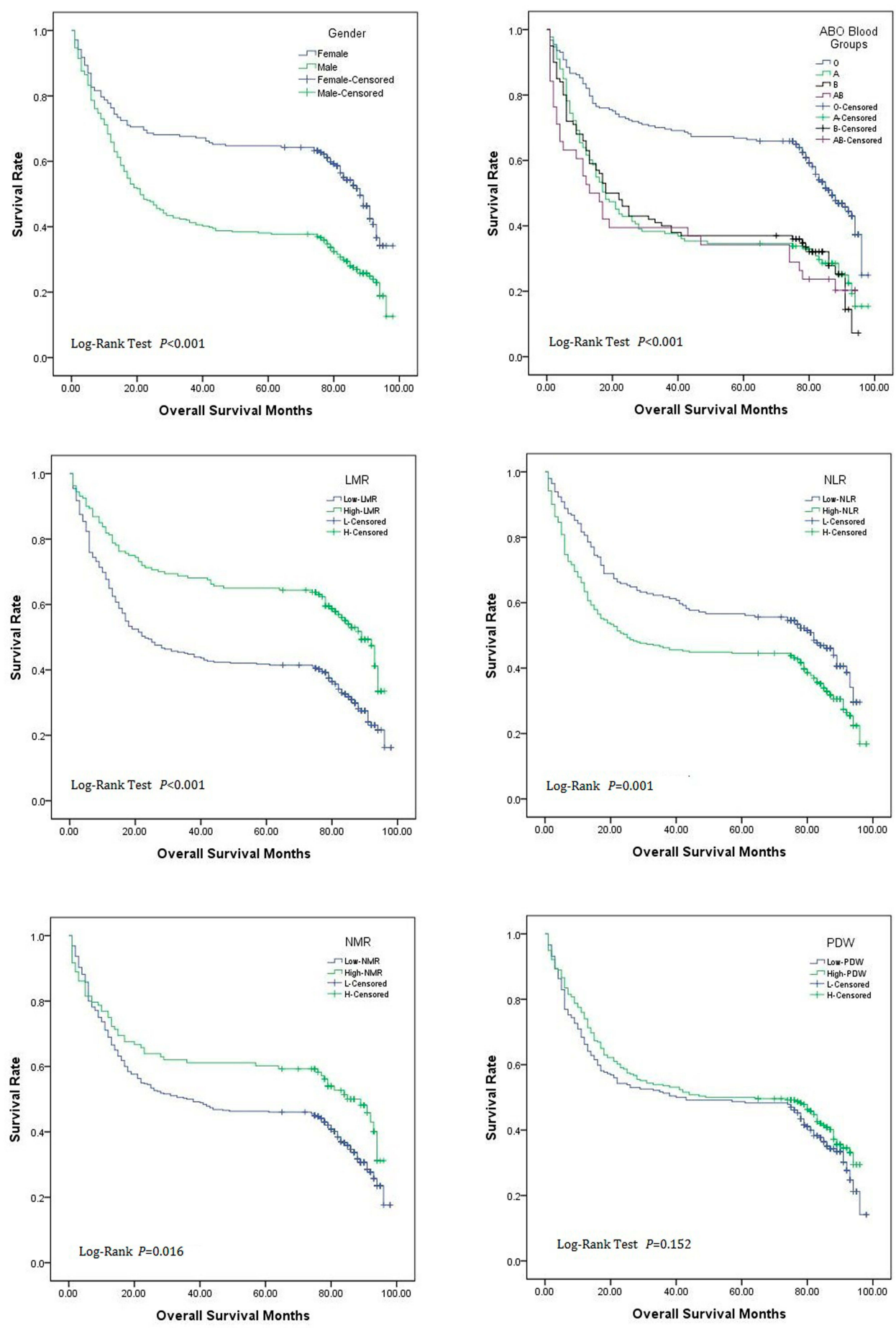

Figure 2 Analysis of survival curve of sex, blood group and meaningful clinical indexes.

and $\mathrm{AB}$ blood patients increased 4.407 times $(95 \% \mathrm{CI}$ 2.871-6.766). When NLR value increased by 1 , the risk of death increased by 1.103 times (95\% CI 1.028-1.183). While PLT and PDW were independent protective factors for the prognosis of gastric cancer, and the risk of death decreased by 0.998 times when PLT increased by 1 (95\%
CI $0.997-1.000)$. The risk of death decreased by 0.891 times (95\% CI 0.797-0.996), when PDW increased by 1.

\section{Discussion}

From the general data of 488 patients, it could be seen that male over 60 years old were more likely to suffer from 
Table 3 Univariate Cox Regression Analysis Results of All Indicators

\begin{tabular}{|l|l|l|l|l|l|}
\hline & $\boldsymbol{\beta}$ & Wald $\chi^{2}$ & $\mathbf{P}$ & HR & $\begin{array}{l}\text { 95\% } \\
\text { Confidence } \\
\text { Interval }\end{array}$ \\
\hline $\begin{array}{l}\text { Gender } \\
\text { (male/ } \\
\text { female) }\end{array}$ & 0.601 & 25.147 & $<0.00 I^{*}$ & 1.825 & $1.443-2.308$ \\
\hline ABO blood type & & & & \\
\hline A/O & 0.701 & 25.454 & $<0.00 I^{*}$ & 2.107 & $1.536-2.648$ \\
B/O & 0.739 & 23.993 & $<0.00 I^{*}$ & 2.094 & $1.558-2.814$ \\
AB/O & 0.864 & 17.623 & $<0.00 I^{*}$ & 2.372 & $1.585-3.550$ \\
Hb & -0.001 & 0.061 & 0.804 & 0.999 & $0.995-1.004$ \\
PLT & -0.001 & 2.403 & 0.121 & 0.999 & $0.998-1.000$ \\
RDW & 0.018 & 1.028 & 0.311 & 1.018 & $0.984-1.054$ \\
PDW & -0.036 & 2.118 & 0.146 & 0.965 & $0.919-1.013$ \\
PLR & -0.005 & 0.653 & 0.419 & 0.995 & $0.983-1.007$ \\
LMR & -0.005 & 0.558 & 0.455 & 0.995 & $0.982-1.008$ \\
NMR & 0.000 & 0.001 & 0.978 & 1.000 & $0.994-1.006$ \\
NLR & 0.060 & 4.796 & $0.029 *$ & 1.062 & $1.006-1.120$ \\
\hline
\end{tabular}

Note: *It has statistical significance.

Table 4 Cox Regression Analysis of Various Indicators

\begin{tabular}{|l|l|l|l|l|l|}
\hline & $\begin{array}{l}\boldsymbol{\beta} \\
\text { Value }\end{array}$ & Wald $\chi^{2}$ & $\mathbf{P}$ & HR & $\begin{array}{l}\text { 95\% } \\
\text { Confidence } \\
\text { Interval }\end{array}$ \\
\hline $\begin{array}{l}\text { Gender } \\
\text { (male/ } \\
\text { female) }\end{array}$ & 1.087 & 61.197 & $<0.00 I^{*}$ & 2.964 & $2.258-3.891$ \\
\hline ABO blood type & & & & \\
\hline A/O & 1.245 & 64.417 & $<0.00 I^{*}$ & 3.472 & $2.562-4.706$ \\
B/O & 1.214 & 56.443 & $<0.00 I^{*}$ & 3.368 & $2.454-4.624$ \\
AB/O & 1.483 & 45.984 & $<0.00 I^{*}$ & 4.407 & $2.871-6.766$ \\
Hb & -0.002 & 0.346 & 0.556 & 0.998 & $0.993-1.004$ \\
PLT & -0.002 & 4.692 & $0.030^{*}$ & 0.998 & $0.997-1.000$ \\
RDW & 0.013 & 0.335 & 0.563 & 1.014 & $0.968-1.061$ \\
PDW & -0.115 & 4.102 & $0.043^{*}$ & 0.891 & $0.797-0.996$ \\
PLR & 0.020 & 1.927 & 0.165 & 1.020 & $0.992-1.049$ \\
LMR & 0.002 & 0.049 & 0.825 & 1.002 & $0.985-1.019$ \\
NMR & -0.001 & 0.066 & 0.798 & 0.999 & $0.989-1.008$ \\
NLR & 0.098 & 7.419 & $0.006^{*}$ & 1.103 & $1.028-1.183$ \\
\hline
\end{tabular}

Note: *It has statistical significance.

gastric cancer, and the infection rate of Helicobacter pylori in patients was as high as about $63.5 \%$. Among the tumor markers commonly used in the laboratory, even the CEA and CA199 tumor markers recommended in the guidelines for the diagnosis and treatment of gastric cancer have a low positive rate in gastric cancer patients, which were consistent with the existing reports. ${ }^{7,8}$ However, the existing reports showed that the incidence of blood group $\mathrm{O}$ was lower than non-O blood group in gastric cancer patients. ${ }^{9,10}$ But this study showed that the proportion of O blood group patients was the highest in 488 patients, which was inconsistent with the previous reports, it may be due to different population selection.

Many reports have shown that LMR, NLR and NMR, as inflammatory indicators, were closely related to the prognosis of various diseases. ${ }^{11,12}$ And could be used as prognostic factors for a variety of malignant tumors. For example, LMR and NLR could be used as prognostic indicators for esophageal cancer patients undergoing concurrent radiotherapy and chemotherapy. ${ }^{13}$ LMR could also be used as prognostic indicators for high-risk thyroid cancer. ${ }^{14,15}$ There were also some reports about the relationship between gastric cancer and these inflammatory indexes. Jinping et al reported that NLR could be used as a prognostic factor in patients with gastric cancer, and preoperative NLR $>3.79$ indicated a poor prognosis in patients with gastric cancer. It was also proved by Shumei et al. ${ }^{16}$ And the research results of Peng ect show that high NLR was an independent risk factor for the prognosis of postoperative cancer patients (HR 1.768-2.174). ${ }^{17}$ And the results of our study were generally in line with the above reports. It indicated that NLR was indeed an independent predictor of the prognosis of gastric cancer. At the same time, PLT and PDW were found to be independent protective factors for the prognosis of patients with gastric cancer. Ding Qunhua reported that reduced PDW before surgery was a poor prognosis factor of gastric cancer, which may become a prognostic indicator of gastric cancer. ${ }^{18}$ It was consistent with the results of this study that proved the important application value of platelet-related indicators in the prognosis of patients with gastric cancer.

ABO blood group system is one of the most widely used blood groups in clinical practice. In the past few years, some studies have reported that there may be a close relationship between $\mathrm{ABO}$ blood group and cancer risk. For example, A-type blood group could increase the incidence of a series of cancers, such as liver cancer, pancreatic cancer, ovarian cancer and nasopharyngeal cancer, and could predict the prognosis of some cancer patients. ${ }^{19-22}$ However, it has been reported that ABO blood group was only related to the occurrence of gastric cancer, and has no correlation with the prognosis of gastric cancer patients. ${ }^{23-25}$ Due to the fact that there was a correlation between $\mathrm{ABO}$ blood group and the 
prognosis of most malignant tumors, this study restudied the correlation between $\mathrm{ABO}$ blood group and gastric cancer. It was found that $\mathrm{ABO}$ blood group was also a good independent prognostic factor for gastric cancer, especially compared to gastric cancer patients with O blood group, and the patients with non-O blood groups had a higher risk of death. The direct biological mechanism of the relationship between ABO blood group and many kinds of tumors, including gastric cancer, was still inconclusive, but some hypotheses could explain this relationship. Firstly, the ABO gene was located on chromosome $9 \mathrm{q}$ and formed from seven exons, which encodes a glycosyltransferase that catalyzes the transfer of carbohydrates and binds to $\mathrm{H}$ antigen to form an antigenic structure of $\mathrm{ABO}$ blood group. Blood group antigens are expressed not only on the surface of red blood cells, but also in many other tissues, it has been proven that the modified expression of blood group antigen on the surface of tumor cells could change the motility, drug resistance, apoptosis and immune escape ability of tumor cells. In addition, because there was a high positive correlation between $\mathrm{AB}$ blood group and the circulating level of soluble intercellular adhesion molecule-1 (sICAM-1), and a high level of sICAM-1 contributes to the immune escape of malignant tumors, patients with O blood group are less likely to have a poor prognosis due to the lack of corresponding blood group antigens. $^{26,27}$ The results of this study on the relationship between $\mathrm{ABO}$ blood group and the prognosis of gastric cancer were contradictory to previous reports, and more studies are needed to verify which conclusion is more correct.

\section{Conclusion}

The related indexes of $\mathrm{CBC}$ and $\mathrm{ABO}$ blood group test, as cheap indexes in clinical laboratory, have a certain significance in judging the prognosis of patients with gastric cancer. However, because the laboratory test results of $\mathrm{CBC}$ related indexes are too uncontrollable factors and the degree of variation of the indexes is too large, the role of $\mathrm{CBC}$ in the judgment of clinical prognosis is limited. While the results of ABO blood group system are constant, more research should be carried out in judging the prognosis of patients with gastric cancer in the future.

\section{Acknowledgments}

The authors are grateful to the hospital medical record department for the data source.

\section{Disclosure}

The authors declare that they have no conflicts of interest.

\section{References}

1. Chen WQ, Zheng RS, BAADE PD, et al. Cancer statistics in China, 2015. CA Cancer J Clin. 2016;66(2):115-132. doi:10.3322/ caac. 21338

2. Weiwei G, Shenglan L, Ruying H, et al. Survival rate of gastric cancer in Zhejiang Province from 2005 to 2010. Chin J Oncol. 2014;36(8):636-639.

3. Peng P, Chunxiao W, Yangming G, et al. Study on the survival rate of gastric cancer in Shanghai population. Chin J Cancer. 2016;26 (5):414-420.

4. Xiaolin C, Guoqiang Y, Jianrong L, et al. The value of preoperative peripheral blood NLR, d-NLR, PLR and LMR in prognostic diagnosis of patients with colorectal cancer. Chin $J$ Immunol. 2015;10:1389-1393.

5. Jinlan H, Shihao Z, Qishui O, et al. Analysis of the value of preoperative peripheral blood NLR, dNLR and LMR in evaluating the prognosis of patients with glioblastoma. J Fujian Med Univ. 2017;51 (6):404-409.

6. Huizi R, Xiping S. Study on the relationship between ABO blood group and 9 kinds of malignant tumors. Gansu Med. 2015;34 (4):241-243.

7. Xiaojuan C, Jing W, Jing $\mathrm{Y}$, et al. The value of abnormal prothrombin combined with CEA, CA72-4 and CA199 in the diagnosis and treatment of gastric cancer. Res Cancer Prev Treat. 2018;45(6):395-399.

8. Liya Z, Shiyu X. Research progress of Helicobacter pylori and gastric cancer. J Clin Intern Med. 2018;35(12):797-799.

9. Minfei P, Sufei Y, Jing W, et al. Wang Donglian. Study on the relationship between $\mathrm{ABO}$ blood group and the risk of 8 kinds of malignant tumors. Chin J Health Inspection. 2014;24(06):811813,823 .

10. Lingjun $\mathrm{X}$, Jixiang Z. ABO blood group distribution and clinical characteristics of gastric cancer patients. Shandong Med. 2012;52 (17): $16-18$.

11. Zhang A, Ning L, Han J, et al. Neutrophil-To-lymphocyte ratio as a potential biomarker of neovascular glaucoma. Ocul Immunol Inflamm. 2019;10:1-8. doi:10.1080/09273948.2019.1677916

12. Lin Y, Peng Y, Chen Y, et al. Association of lymphocyte to monocyte ratio and risk of in-hospital mortality in patients with acute type A aortic dissection. Biomark Med. 2019;13(15):1263-1272. doi:10. 2217/bmm-2018-0423

13. Li KJ, Xia XF, Su M, et al. Predictive value of lymphocyte-to-monocyte ratio (LMR) and neutrophil-to-lymphocyte ratio (NLR) in patients with oesophageal cancer undergoing concurrent chemoradiotherapy. $B M C$ Cancer. 2019;19(1):1004. doi:10.1186/s12885-019-6157-4

14. Song L, Zhu J, Li Z, et al. The prognostic value of the lymphocyte-to-monocyte ratio for high-risk papillary thyroid carcinoma. Cancer Manag Res. 2019;11:8451-8462. doi:10.2147/ CMAR.S219163

15. Jinping M, Zhi W, Jianwei L, et al. The application of neutrophil/ lymphocyte ratio in the prognosis of gastric cancer. Chin J Gastrointest Surg. 2011;14(12):944-947.

16. Shumei L, Xiaoli X, Di L, et al. The prognostic value of peripheral blood neutrophil to lymphocyte ratio and platelet to lymphocyte ratio in patients with gastric cancer. Chin J Oncol. 2014;36(12):910-915.

17. Peng S, Zhao Y, Xin D, et al. The clinical significance of preoperative neutrophil to lymphocyte ratio (NLR) in predicting the prognosis of gastric cancer. Mod Oncol. 2012;20(5):987-991.

18. Qunhua D, Wei H, Zhen X, et al. Relationship between preoperative platelet distribution width and clinicopathological characteristics and prognosis in patients with gastric cancer. Chin Oncol Clin. 2018;45 (2):72-76 
19. Li Q, Yu CH, Yu JH, et al. ABO blood group and the risk of hepatocellular carcinoma: a case-control study in patients with chronic hepatitis B. PLoS One. 2012;7:e29928. doi:10.1371/journal. pone.0029928

20. Wang DS, Chen DL, Ren C, et al. ABO blood group, hepatitis B viral infection and risk of pancreatic cancer. Int $J$ Cancer. 2012;131:461-468. doi:10.1002/ijc.26376

21. Poole EM, Gates MA, High BA, et al. ABO blood group and risk of epithelial ovarian cancer within the Ovarian Cancer Association Consortium. Cancer Causes Control. 2012;23:1805-1810. doi:10.1007/s10552-012-0059-y

22. Wang DS, Wang ZQ, Zhang L, et al. Are risk factors associated with outcomes in pancreatic cancer. PLoS One. 2012;7:e41984 doi:10.1371/journal.pone.0041984

23. Wang Z, Liu L, Ji J, et al. ABO blood group system and gastric cancer: a case-control study and meta-analysis. Int $\mathrm{J} \mathrm{Mol} \mathrm{Sci}$. 2012;13:13308-13321. doi:10.3390/ijms131013308
24. Qiu MZ, Zhang DS, Ruan DY, et al. A relationship between ABO blood groups and clinicopathologic characteristics of patients with gastric adenocarcinoma in China. Med Oncol. 2011;28(Suppl 1): S268-73. doi:10.1007/s12032-010-9735-5

25. Xiao S, Feng F, Sun L, et al. Study on the relationship between ABO blood group and prognosis of patients with gastric cancer. Chin $J$ Gastrointest Surg. 2015;18(10):1011-1015.

26. Kayser K, Hauck E, Andre S, et al. Expression of endogenous lectins (galectins, receptors for ABH-epitopes) and the MIB-1 antigen in esophageal carcinomas and their syntactic structure analysis in relation to post-surgical tumor stage and lymph node involvement. Anticancer Res. 2001;21:1439-1444.

27. Barbalic M, Dupuis J, Dehghan A, et al. Large-scale genomic studies reveal central role of ABO in SP-selectin and sICAM-1 levels. Hum Mol Genet. 2010;19:1863-1872. doi:10.1093/hmg/ddq061

\section{Publish your work in this journal}

OncoTargets and Therapy is an international, peer-reviewed, open access journal focusing on the pathological basis of all cancers, potential targets for therapy and treatment protocols employed to improve the management of cancer patients. The journal also focuses on the impact of management programs and new therapeutic agents and protocols on patient perspectives such as quality of life, adherence and satisfaction. The manuscript management system is completely online and includes a very quick and fair peer-review system, which is all easy to use. Visit http://www.dovepress.com/ testimonials.php to read real quotes from published authors. 